\title{
Aneurysmal bone cyst of the spine: Nine pediatric case report
}

\author{
Walid Osman ${ }^{1}$, Lassaad Hassini ${ }^{1}$, Zeineb Alaya ${ }^{2}$, Mohamed A Khalifa ${ }^{1}$, Mourad Mtaoumi ${ }^{1}$, Karim Bouattour ${ }^{1}$ and Mohamed B Ayèche $^{1}$ \\ ${ }^{1}$ Department of Orthopaedics, Sahloul Hospital, Faculty of Medicine of Sousse, Sousse 4000, Tunisia \\ ${ }^{2}$ Department of Internal Medicine, Mohamed Tahar Maamouri Hospital, 8000 Nabeul, Tunisia
}

\begin{abstract}
Purpose: Aneurysmal bone cysts are benign and expansile osteolytic lesions that can occur in any location in the spine. This report describes clinical characteristics and treatment results of 9 young patients with aneurysmal bone cyst of the spine.

Methods: Between 1997 and 2015,9 young patients with aneurysmal bone cyst of the spine were surgically treated in our department. The clinical records, radiographs, and operative reports were analysed.

Results: This study included 9 paediatric cases ( 6 girls, 3 boys) managed for aneurysmal bone cyst. The mean age was 11 years. Posterior spinal elements were affected in all cases. The lesion affected the cervical spine (1 case), thoracic spine (3 cases), and lumbar region (5 cases). The radiological aspect was typical in all cases. MRI imaging was very suggestive of the diagnosis showing specific features of the lesion. It was described as an expansil lytic process arising from vertebral posterior elements. All patients underwent a surgical removal of the tumor following a surgical biopsy. A segmental instrumented fusion in order to establish a proper spinal stability was made in 5 cases. Histological report confirmed the diagnosis of aneurysmal cyst in all patients. The mean follow-up was 7,7 years. The follow-up course was uneventful.
\end{abstract}

Conclusion: The presented study demonstrates that lesional resection and structural spinal integrity made by local spinal fusion allow good outcomes with a low rate of complications and low recurrence.

\section{Introduction}

Aneurysmal bone cysts $(\mathrm{ABC})$ are locally aggressive lesions and constituting $1-5 \%$ of primary bone tumors. The lesion can occur in any location in the spine. Children and young adults are often affected by this entity. There is not specific clinical presentation for this entity. The management of such tumors remains controversial. Thus, a complete excision of the lesion can be associated with high morbidity related to the destructive nature of the tumor. The challenge is to establish a suitable spinal stability following the tumor removal. The main goal of the present study was to highlight the potentially devastating effects that can be caused by benign tumors in the vertebral column and to demonstrate the role of MRI (magnetic resonance imaging) in a detailed description of the lesion leading to suggest properly the diagnosis before the operation.

\section{Patients and methods}

We report our experience concerning the management of aneurysmal bone cyst of the spine in children. Charts of 9 cases dealing with aneurysmal bone cyst affecting the spine were retrospectively reviewed. The study was conducted in the department of orthopaedic surgery in university hospital (Sahloul, Sousse). Over 20 years of study period (from January 1997 to August 2015), data were collected using case records of patients that were gathered from medical observations, surgical and histological reports. The mean follow-up was 7,7 years.

\section{Results}

A single-center retrospective study covered the period 19972016. Nine children were included (data and patients demographic characteristics are detailed), 6 females, 3 males were reviewed. Mean age: 11 years (range: 5-18 years). Mean follow-up was 7,7 years (range: 2-12 years). Back pain is the most revealing symptom. The pain is located in the dorsal segment. The posterior elements of the spine and pedicles are affected in all cases with an extension into the vertebral bodies, neural foramina and spinal canal in 3 cases. Neurological symptoms were documented in 3 cases (Paraparesis: 2 cases, lumbosacral radiculopathy: 1 case). A tender para-vertebral mass was found in 2 cases on physical examination. Biological tests were within normal limits. Several segments of the spine were affected: cervical spine ( 1 cases), thoracic spine (3 cases), and lumbar spine (5 cases). Preoperative imaging comprised plain radiographs, CT/ scan and MRI of the spine showing a lytic process arising essentially from the posterior elements of the spine. Multiple fluid levels with intralesional septations was the most characteristic features. An initial surgical biopsy was done in all patients leading to the diagnosis of aneurysmal bone cyst of the spine.

A completed surgical excision of the bony lesion was carried-out in all patients. Spinal stability was established by instrumental fusion

${ }^{\star}$ Correspondence to: Zeineb Alaya, Department of Internal Medicine, Mohamed Tahar Maamouri Hospital, 8000 Nabeul, Tunisia, E-mail: zeineb_a@hotmail.fr

Key words: bone cysts, aneurysmal, pediatrics, spine

Received: October 19, 2020; Accepted: December 09, 2020; Published: December 31, 2020 
in 5 patients, an emergent decompressive laminectomy with surgical excision was carried out in 2 patients who presented with neurological symptoms due to an acute spinal cord compression (case 5 and 8). The histo-pathological report was consistent with the diagnosis of aneurysmal bone cyst of the spine. The mean follow-up was 7,7 years. There was a full recovery without neurological deficit or evidence of recurrence on post-operative radiological assessment.

\section{Case presentation}

\section{Case 1}

A 5-year-old girl with unremarkable medical or surgical history, presented with 2-month history of posterior neck pain following a benign trauma. The pain was getting progressively worse and did not relieve under symptomatic treatment. She denied any radicular symptoms, bowel, or bladder incontinence. On physical examination, she was afebrile, the posterior cervical region was tender on palpation. Higher mental functions and cranial nerve examination were normal. Motor and sensory examination in the lower limbs was normal, she had no pathologic reflexes. Biologic parameters were within normal limits. Plain radiographs of the cervical spine showed a lytic process in the $\mathrm{C} 2$ spinous process (Figure 1). A technetium bone scan showed an increased uptake in the posterior aspect of $\mathrm{C} 2$. These were followed up by computerized tomography (Figure 2) which demonstrated an heterogeneous lytic lesion involving the $\mathrm{C} 2$, right pedicle and rightsided lamina with direct spine cord compression. Magnetic resonance imaging (Figure 3) revealed a multilocular cystic bony process arising from the posterior aspect of $\mathrm{C} 2$ with intact cortex and fluid-fluid levels. The lesion did not involve the spinal canal. A surgical biopsy was consistent with an $\mathrm{ABC}$. A completed surgical excision of the bony lesion with $\mathrm{C} 2-\mathrm{C} 3$ posterior fusion was carried-out. The histopathological report confirmed the diagnosis of aneurysmal bone cyst of the spine. The follow up period was of 10 years. There was a full recovery without neurological deficit or evidence of recurrence on follow-up radiographs (Figure 4).

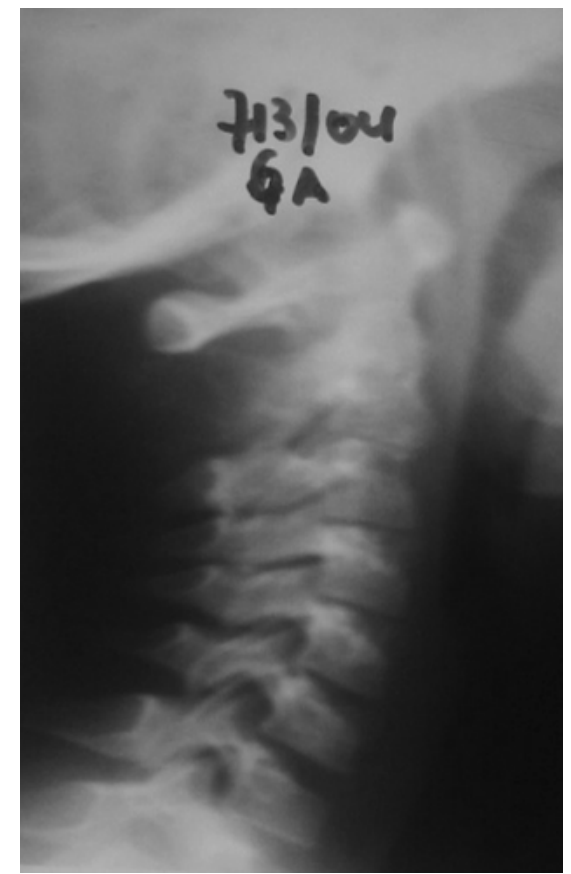

Figure 1. Pre-operative lateral cervical spine radiograph showing a lytic process involving the $\mathrm{C} 2$ spinous process

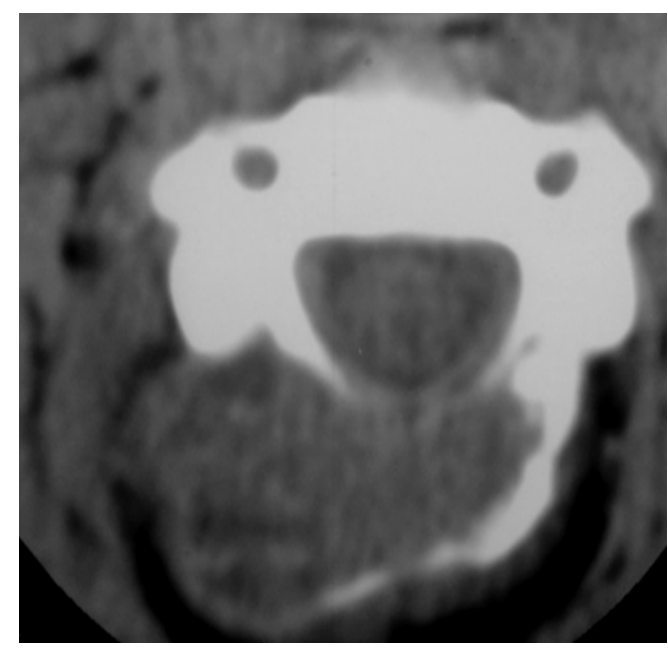

Figure 2. Pre-operative axial CT scan: presence of an expansile heterogeneous tissue process blowing the posterior arch of $\mathrm{C} 2$

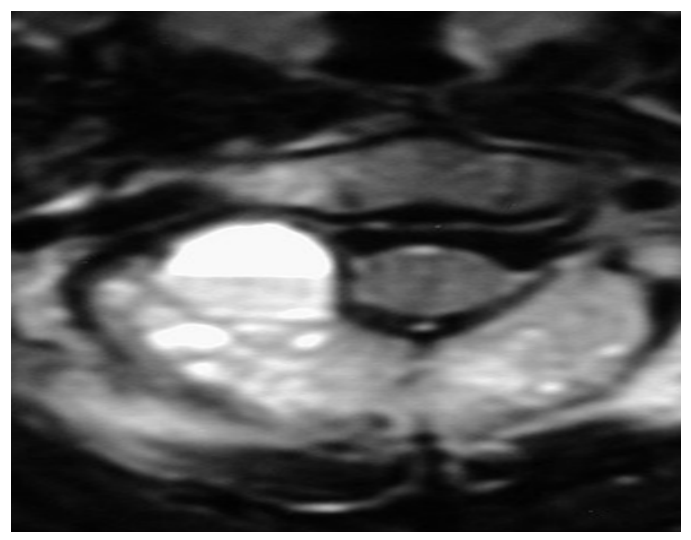

Figure 3. Pre-operative MRI of the neck: multilocular cystic bony process with fuid-fluid levels

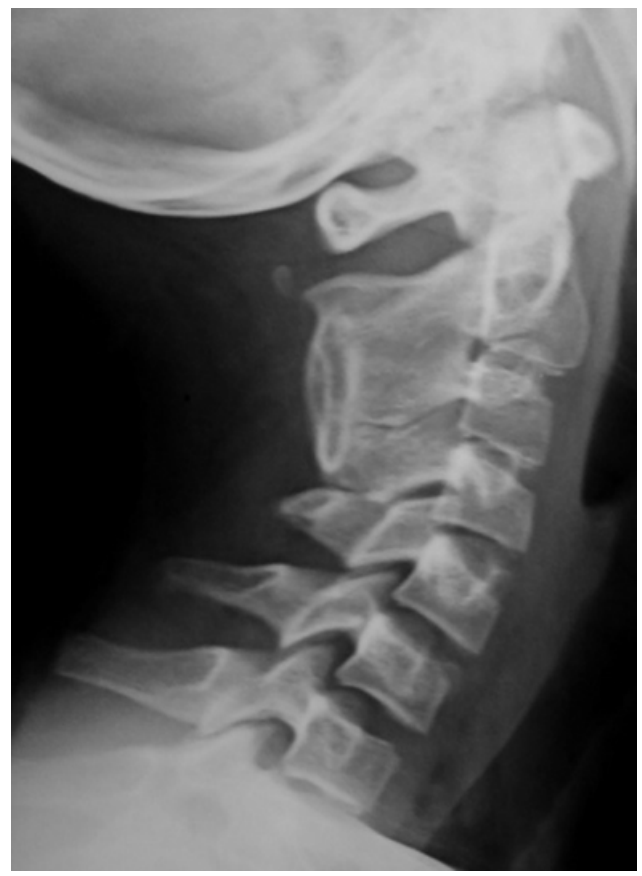

Figure 4. Lateral cervical spine radiograph at last follow-up showing bone filling of the lesion and $\mathrm{C} 2$ to $\mathrm{C} 3$ fusion. 


\section{Case 2}

A 15-year-old girl presented complaining of a 5-month history of low back pain. There was no history of trauma and she was otherwise well without any particular radiation. She denied any radicular symptoms. Physical examination revealed swelling in the lumbar para vertebral region, she had no cutaneous abnormalities nor palpatory mass and was neurologically intact. Biologic parameters were within normal limits. Plain radiographs of lumbar spine showed a lytic defect in the L4 spinous process and lamina, there was no evidence of cortical breach. A technicium bone scan revealed a mild increased uptake in L4 vertebra. The following computerized tomography revealed a lytic process arising from the left lamina of L4 vertebra (Figure 1) and extending to the vertebral body and transverse process with intact cortical rims and matrix density. There was a peripheral enhancement after gadolinium administration. Magnetic resonance imaging demonstrated an expansile process involving L4 vertebra and affecting the transverse process, left pedicle and the vertebral body. The lesion was intermediate signal on T1-weighted images and increased signal uptake on T2-weihted images. There were multiple enhanced septations after gadolinium administration (Figure 2). On the basis of these specific radiological findings, the diagnosis an aneurysmal bone cyst was suspected. The patient was operated, she carried out a surgical biopsy confirming the diagnosis of $\mathrm{ABC}$ followed by a surgical excision of the lesion within the lamina, the left pedicle and a part of the vertebral body. A segmental instrumented fusion (Figure 3) was made. The histopathology was consistent with an aneurismal bone cyst. There was a full recovery, and the patient was asymptomatic at the last control with MRI showing no evidence of recurrence (Figure 4).

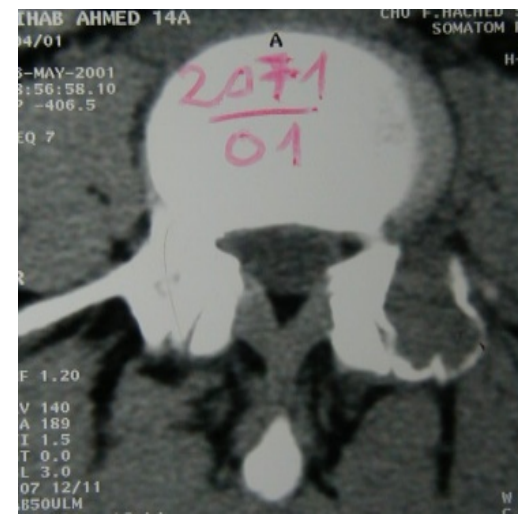

Figure 1. Computerized tomography revealing a lytic process arising from the left lamina of L4 vertebra
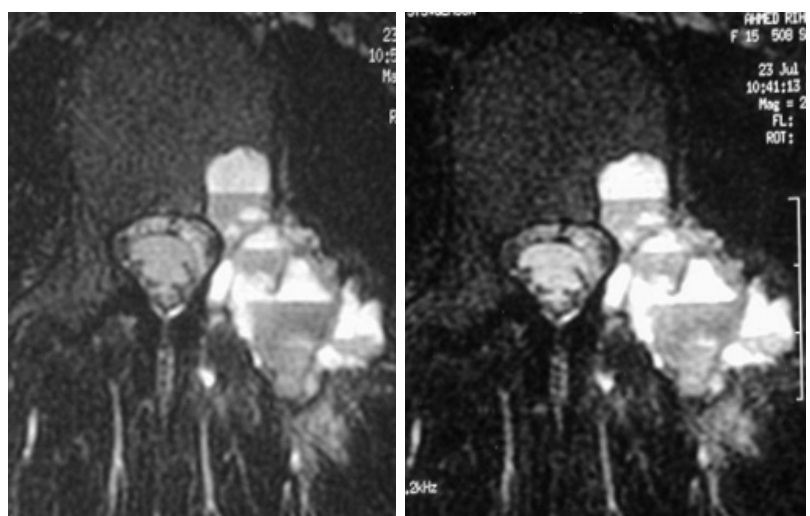

Figure 2. MRI imaging showing the lesion with multiple enhanced septations and fluidfluid levels

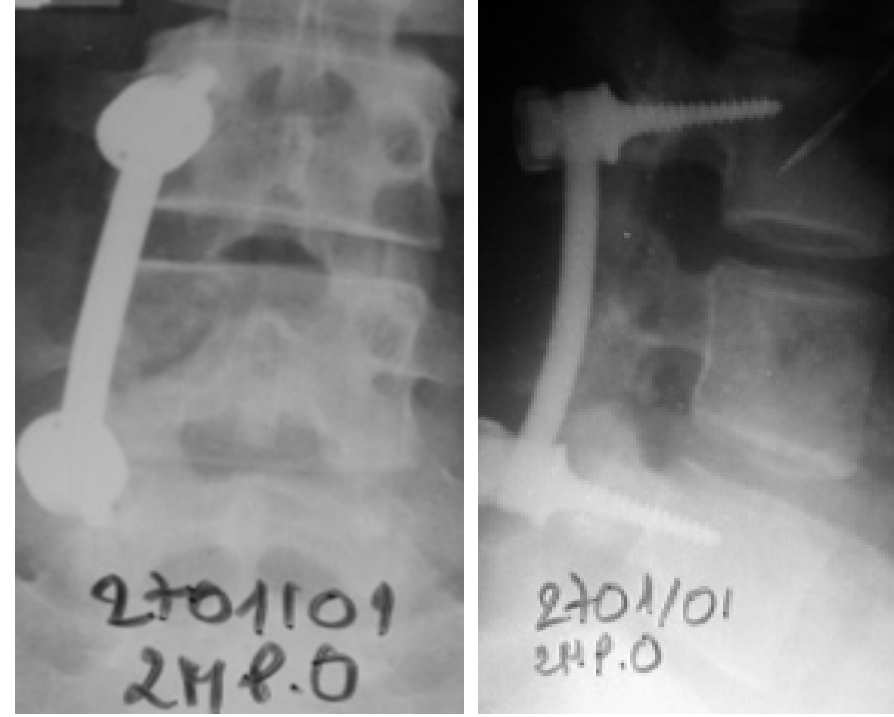

Figure 3. Plain radiographs demonstrating the spinal stability obtained by surgica instrumentation
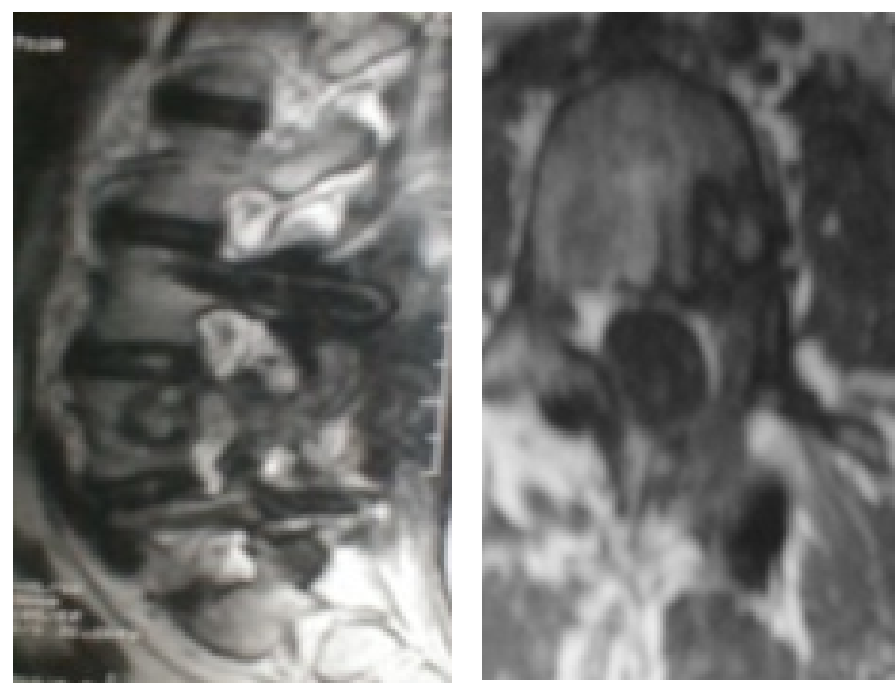

Figure 4. Last control of 4 years. MRI showing no evidence of recurrence

\section{Case 3}

An 11-year-old boy previously fit and well presented with back pain and spinal deformation. There was a history of trauma and symptoms settled 2 months after. On examination, movements were restricted in flexion and extension (Figure 1). Plain radiographs (Figure 2) showed a lytic defect involving T11 vertebral pedicle. Tomographic images revealed a pathological T11 vertebra with several abnormalities in the posterior elements secondary to an expansile lesion.

Magnetic images (Figure 3) were compatible with an expansile cystic lesion arising from posterior aspect of T11 vertebra and extending into the bony canal and costo vertebral joint. These radiological findings were highly suggestive of aneurysmal bone cyst. The patient underwent a surgical decompressive left laminectomy of T11 with excision of the lesion. Spinal stability (Figures 4 and 5) was established by instrumentation from T9 to L1 and iliac crest bone grafting. Histological report confirmed the diagnosis. The follow-up was 9 years, the patient was symptoms free with no recurrence. 

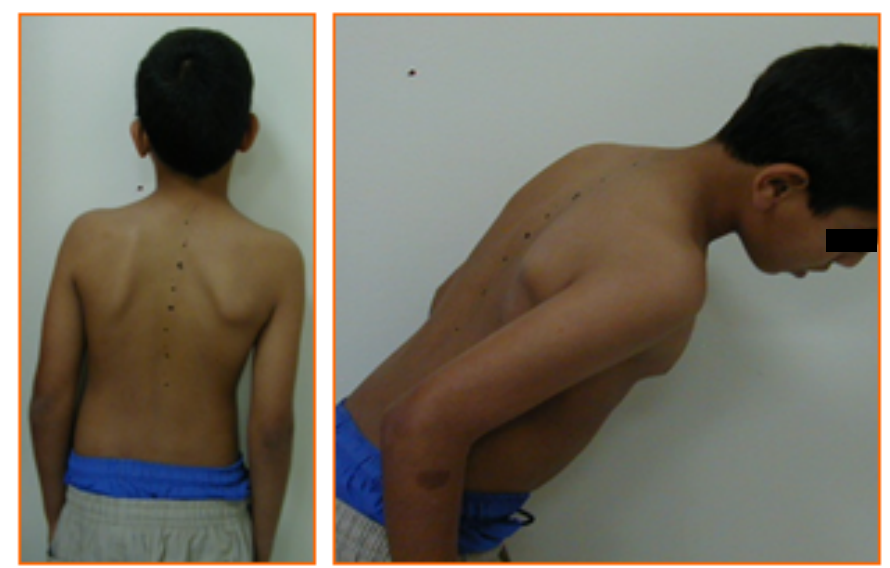

Figure 1. The patient stood with a spinal deformation and limited range of motion in flexion and extension

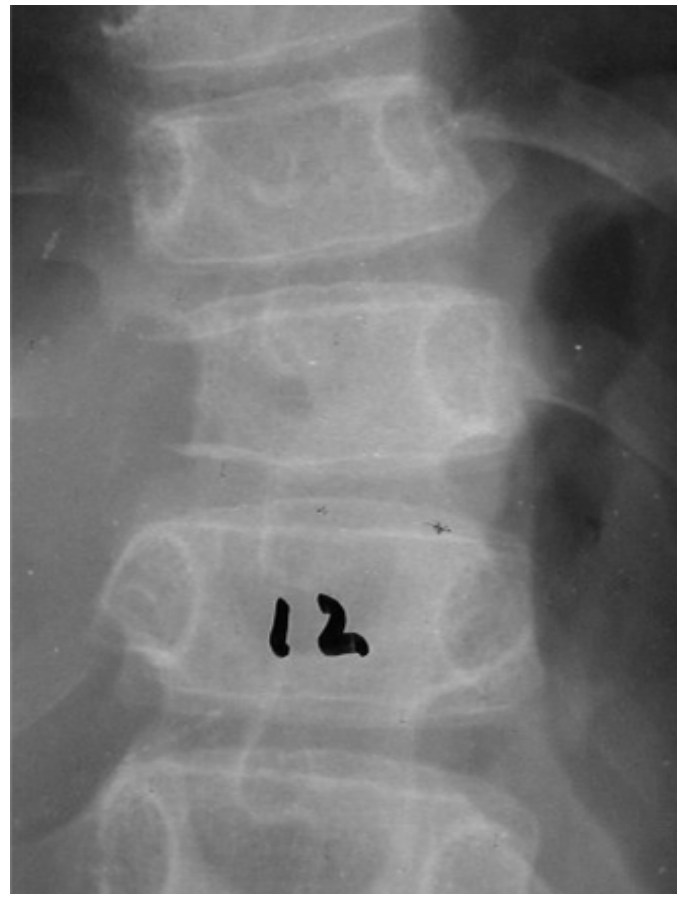

Figure 2. Plain radiograph showing a lytic defect involving T11 vertebral pedicle
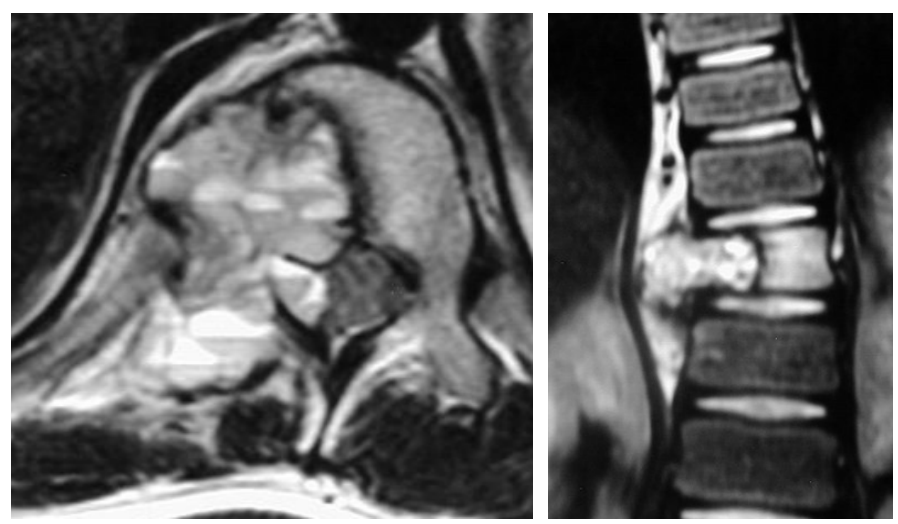

Figure 3. MRI images revealing an expansile cystic lesion with multiple fluid-fluid levels arising from posterior aspect of T11 vertebra and extending into the bony canal and costo vertebral joint

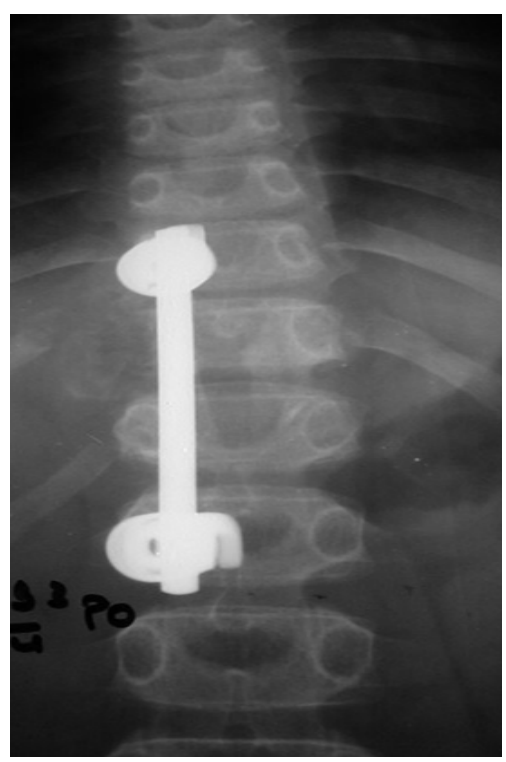

Figure 4. Spinal stability was established by instrumentation from T9 to L1 and iliac crest bone grafting

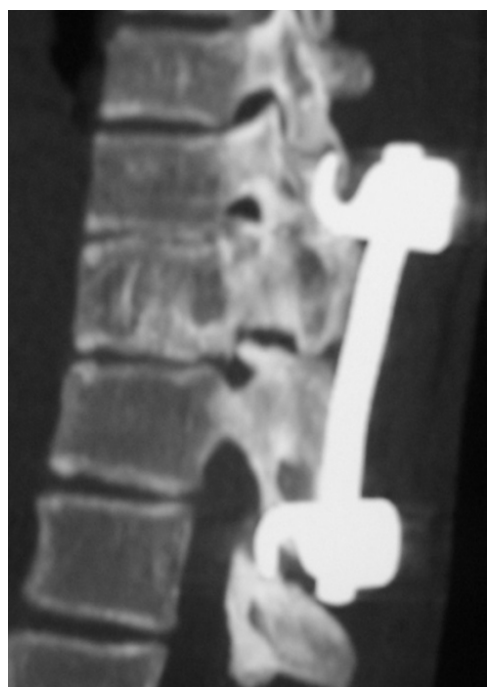

Figure 5. Parasagittal CT-scan reconstruction at final follow-up showing the bony filing and fusion of arthrodesis

\section{Case 4}

A 7-year-old boy, previously healthy presented to our department with a 6-month- history of right flank pain. Pain was partially relieved by rest and anti-inflammatory drugs. Physical examination was irrelevant except a palpatory tenderness on D9 spinous process. Motor and sensory functions were preserved. Laboratory investigations were within normal range. CT-scan and MRI showed a large lytic and expansile lesion arising from the posterior arch of T9 and extending intraspinal with compression of the spinal cord (Figure 1). That osteolytic mass involved the right posterior vertebral body and was characterized on imaging by fluid-fluid levels delineated by thin septa and was significantly enhanced after contrast injection (Figures 2 and 3). A surgical biopsy was done confirming the diagnosis followed by a complete surgical removal of the process with decompression of the spinal cord. Histopathological report was consistent with an aneurysmal cyst. The patient presented at the last follow-up ( 1 year post operatively) with full recovery and no evidence of recurrence. 


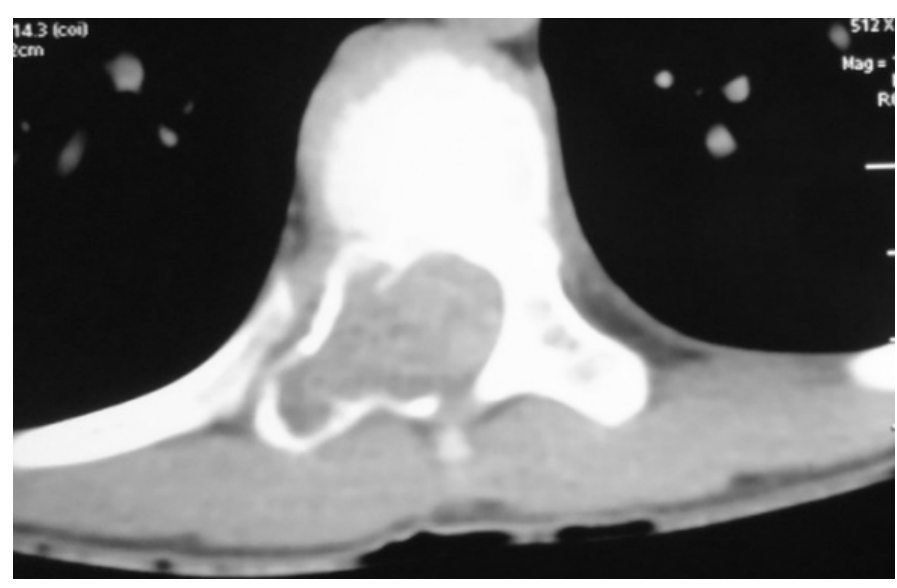

Figure 1. CT scan showing a multilocular lytic lesion involving the posterior arch vertebral body of T9
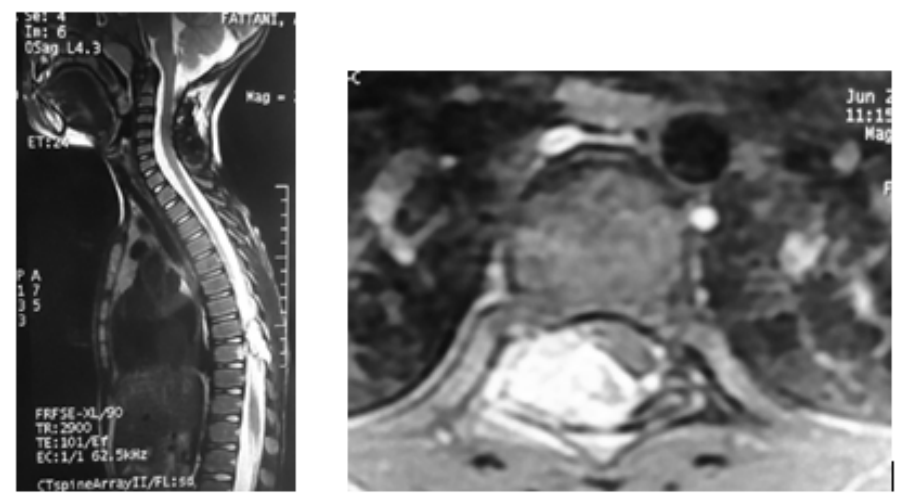

Figure 2. a and b: Sagittal and axial MRI demonstrating an expansile osteolytic lesion arising from the posterior arch of T9 and involving the right posterior vertebral body. It is hyperintense T2-weighted signals with fluid-fluid levels delineated by thin septa

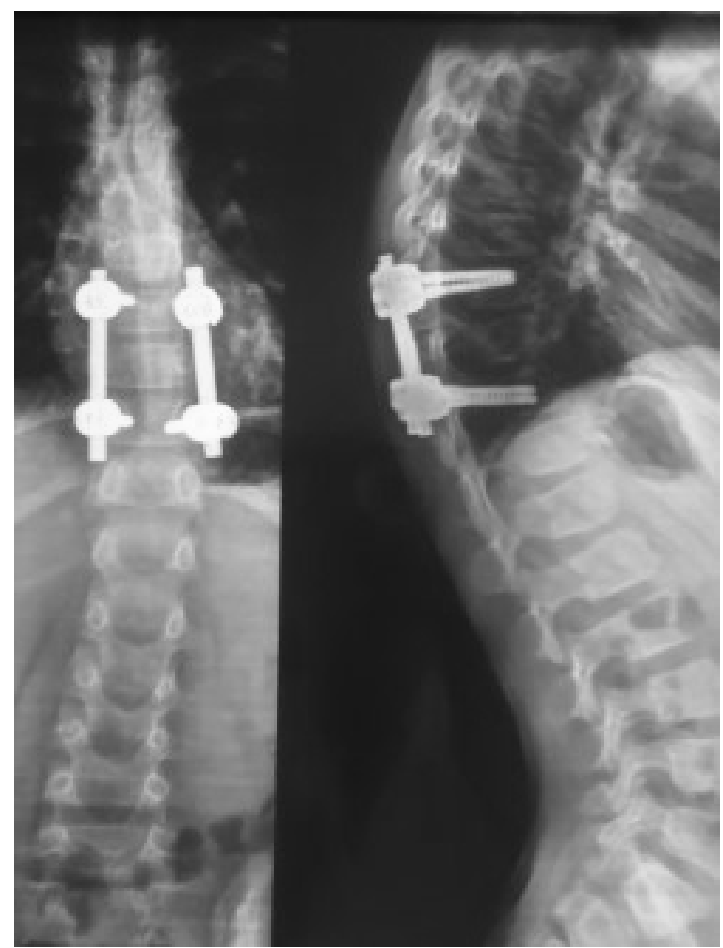

Figure 3. Post-operative plain radiographs demonstrating a segmental instrumented fusion

\section{Discussion}

Aneurysmal bone cysts are benign lesions that represent less than $1 \%$ of solid bone tumors with $8 \%$ to $30 \%$ of $\mathrm{ABCs}$ arising in the spine [1]. ABCs can involve any part of the skeleton, but especially the long bones (67\% of cases), spine (15\%) and pelvis (9\%). The metaphysisis most often involved, and the bones most frequently involved are the distal femur, tibia, humerus and fibula. $\mathrm{ABC}$ of the spine is rarely reported, and it usually involves the posterior arch, mainly of the lumbar, followed by cervical and then thoracic vertebrae [2]. The lumbar location was predominantly affected in the present study (5/9 cases). Most commonly, this condition is essentially reported in patients younger than 20 years and it has been reported to predominantly affect females $[1,3]$.

The exact origin of $\mathrm{ABC}$ is still unclear. Despite being a benign entity, there is ongoing debate about the possible neoplastic nature of those tumors. Kenney et al. [4] have assessed cytogenetically many of those lesions and found the presence of translocation on chromosome 17, which might lead to a neoplastic lesion later on. Thus, such interesting phenomenon might raise new questions about the real nature of such tumors and require cytogenetic studies in patients with $\mathrm{ABC}$ to distinguish those with benign from those with neoplastic origin [5].

$\mathrm{ABC}$ is often revealed by pain, sometimes by swelling, and more rarely by fracture [6,7]. Spinal lesions may be revealed by pain, torticollis, stiff and painful scoliosis, or more rarely a mass, fracture or neurologic symptoms $[2,8,9]$. Clinical findings of spinal $A B C$ are usually nonspecific, such as diffuse pain accompanied by stiffness in the back. The initial presentation is due to neurological symptoms in $60-70 \%$ of cases [10]. A palpable mass or backache that is aggravated in the supine position alerts the physician to this problem. Depending on the level of involvement and the extent of spinal cord compression, a wide variety of neurological symptoms and signs may be noted later, ranging from mild radiculopathy to complete paraplegia or tetraplegia. Acute spinal cord compression can occur in the absence of vertebral body collapse if there is a break in the posterior cortex of the body that results in epidural extension of the lesion [11]. Other signs include scoliosis due to back pain and muscle spasm, paresthesia, and weakness. Scoliosis and kyphosis have been noted in roughly $10-15 \%$ of cases $[12,13]$. In our study, back pain was the most common revealing symptom. It was encountered in all cases. Neurological symptoms were reported in 3 cases; (paraparesis: 2 cases, lumbosacral radiculopathy: 1case).

Extensive preoperative planning with use of advanced imaging to determine the extent of the lesion is one of the important steps in the management of $\mathrm{ABC}$ of the spine in children. X-ray often fails to establish diagnosis, and complementary imaging is required. It shows as an excentric, osteolytic, expansive and sometimes trabeculated lesion containing fine-walled cystic cavities. Internal contours are well defined, with or without an osseous sclerotic ring, and the cortical bone bulges. Loss of cortical contours or extension into soft tissue may mimic a malignant lesion, indicating an aggressive form [14]. Vertebral lesions involve the posterior arch or both the body and the posterior arch $[6,9]$. Computed tomography (CT) is less sensitive than magnetic resonance imaging (MRI) and shows fluid levels in only a third of cases, but it is helpful to assesses the extension of the expansile lytic osseous lesion. MRI is the imaging of choice to complement CT. The typical aspect is an expansive, lesion, lobular or with septa. Multiple fluid levels may be detected on T2-weighted axial sequences at rest, while not specific, they are highly suggestive. Gadolinium injection shows enhancement of the cyst walls and internal septa. 
On sagittal and axial images, it can also obviously show the epidural extension of the mass and its compressive effect on the dural sac and thoracic spinal cord [2]. In the present study, the preoperative MRI indicated an aggressive osteolytic lesion mainly arising from the vertebral posterior arch with fluid-fluid levels delineated by thin septa and infiltration of the surrounding soft tissue. On sagittal and axial images, it showed the epidural extension of the mass and its compressive effect on the spinal cord.

The differential diagnosis must rule out both other benign spine tumors and malignant tumors. In increasing order of frequency, bone tumors in children are eosinophilic granulomas, osteoblastomas, and osteoid osteoma $[13,15]$.

The differential diagnosis includes simple bone cyst, reparative giant cell granuloma, hyperparathyroidism brown tumor, giant cell tumor and malignant primary tumors such as chondrosarcoma, osteosarcoma and Ewing's sarcoma [13]. Therefore, in our patients a surgical biopsy of the lesion was previously done to establish the diagnosis with certainty before the surgery. Microscopically, the composition of the cyst may be dense and cellular, containing plump stromal cells, multinuclear giant cells, and thin-walled blood vessels, or be a preponderantly fibrous tissue with enlarging vascular spaces $[13,16]$.

Conventional treatment has been directed at the surgical removal of either the entire lesion or as much of it as possible. Curettage and bone grafting, with more aggressive treatment reserved for large lesions, or those compressing the spinal cord or nerve roots, and for recurrences. Treatment must be directed toward the most aggressive component present. Stabilization with osteosynthesis is usually performed next. Nevertheless, in young patients it is important to preserve the alignment of the spine and permit harmonious development until growth terminates [17].

Recently, embolotherapy has been used to treat vascular bone tumors to limit blood loss at surgery or as definitive therapy when surgery is not feasible. The goal of embolization is occlusion of the lesion's vascular supply without interfering with the vascularity of surrounding tissue or structures. Successful embolization of an ABC will result in progressive ossification within 2-4 months of the initial embolization, almost always beginning at the margins. Complete ossification may require 8-12 months or longer. The effect of embotherapy is therefore unpredictable. Moreover, potential complications include the occlusion of normal adjacent tissue, iatrogenic pulmonary emboli and catheterrelated complications. We did not use embotherapy, due to the danger of interference with the blood supply to the spinal cord. The addition of postoperative radiotherapy not exceeding 2000 rads has been proposed if some infiltrative tissue remains after partial excision of a huge tumor. Radiation-induced complications include growth plate disturbance, myelopathy, gonadal damage, and sarcomatous changes [18].

Moreover, Intralesional injection of thrombogenic agents (EthiblocW, or alcohol) and calcitonin into spinal ABCs has been reported, but further clinical experience is necessary to establish their efficacy [2].

Complete surgical removal of the tumor is the most accepted treatment modality of spinal $\mathrm{ABC}$, especially in the presence of neurological deficit and impending spinal instability [19]. Reconstruction of the spine with fixation and arthrodesis should be considered when more than one facet joint is resected.

The surgical approach chosen is important, and wide surgical exposure that allows adequate visualization for complete excision of the tumor is advised. A single posterior approach may not be sufficient for complete removal of the lesion with extensive involvement of the vertebral body anterior to the pedicle-body junction [20].

No treatments, other than wide resection, guarantee cure. Other methods show failure rates of 15 to $30 \%$, so that the least aggressive techniques are implemented first, sometimes associated to osteosynthesis in case of severe fragility. Intracystic injection of Ethibloc was reported to provide a 70 to $94 \%$ rate of cure. Ethibloc, however, is no longer avail-able, and other techniques have proved less dangerous. In 2010, Varshney [21] reported a randomized study in which polido-canol (Aetoxisclerol ${ }^{\circ}$ ) sclerotherapy showed success comparable to surgery, with fewer complications.

Surgery was classically the treatment of choice in ABC. Wide resection guarantees against local recurrence, but at the cost of reconstruction problems and of possible complications that the benign nature of $\mathrm{ABC}$ cannot justify. Marginal resection may still be used in very expansive forms. In less aggressive forms, subperiosteal resection limits the risk of local recurrence compared to simple curettage, and periosteal conservation facilitates reconstruction. If the tumor leaves a continuous boney wall curettage and filling by graft, cement, or bone substitute also pro-vides good results, but with a 10 to $30 \%$ risk of local recurrence. Percutaneous curettage and cyst aspiration without filling provide results comparable to intracyst resection [2].

Surgical approach based on a complete removal of the tumor with instrumental arthrodesis is preferable in our team. Satisfactory evolution was obtained in all cases. However other alternatives mainly sclerotherapy can be useful in aggressive forms, where it may be the only option [21].

\section{Conclusion}

$\mathrm{ABCs}$ of the spine present challenges due to their highly vascular nature and the risk of spinal destabilization with resultant neurological compromise. Careful surgical technique and patient specific evaluation must be implemented to minimize morbidity and recurrence. The presented study demonstrates that lesional resection and structural spinal integrity made by local spinal fusion allow good outcomes with a low rate of complications and low recurrence.

\section{Competing interests}

The author(s) declare that they have no competing interests.

\section{References}

1. Ramme AJ, Smucker JD (2011) Balancing spinal stability and future mobility in the cervical spine: surgical treatment of a case of osteoblastoma with secondary aneurysmal bone cyst. Spine J 11: 5-12. [Crossref]

2. Mascarda E, Gomez-Brouchet A, Lambot K (2015) Bone cysts: Unicameral and aneurysmal bone cyst. Orthop Traumatol Surg Res 101: 119-127. [Crossref]

3. Saccomanni B (2008) Aneurysmal bone cyst of spine: a review of literature. Arch Orthop Trauma Surg 128: 1145-1147. [Crossref]

4. Kenney B, Richkind KE, Zambrano E (2007) Solid variant of aneurysmal bone cyst with a novel (X;9) translocation. Cancer Genet Cytogenet 178: 155-159. [Crossref]

5. Termos S, Alsaleh A, Rifaii H, Hammoud-Termos N, Aoun B (2016) Huge aneurysmal bone cyst mimicking a kidney tumor: Case report and review of literature. Int $J$ of Surgery Open 2: 19-21.

6. Ramirez A, Abril JC, Touza A (2012) Unicameral bone cyst: radiographic assessmentof venous outflow by cystography as a prognostic index. J Pediatr Orthop B 21: 489-494. [Crossref]

7. Cottalorda J, Bourelle S (2007) Aneurysmal bone cyst in 2006. Rev Chir Orthop Reparatrice Appar Mot 93: 5-16. [Crossref] 
8. Boriani S, De Iure F, Campanacci L, Gasbarrini A, Bandiera S, et al. (2001) Aneurysmal bone cyst of the mobile spine: report on 41 cases. Eur Spine J 26: 27-35. [Crossref]

9. Amendola L, Simonetti L, Simoes CE, Bandiera S, De Iure F, et al. (2013) Aneurysmal bone cyst of the mobile spine: the therapeuticrole of embolization. Eur Spine $J 22$ : 533-541. [Crossref]

10. Codd PJ, Riesenburger RI, Klimo PJr, Slotkin JR, Smith ER (2006) Vertebra plana due to an aneurysmal bone cyst of the lumbar spine Case report and review of the literature. J Neurosurg 105: 490-495. [Crossref]

11. Liu JK, Brockmeyer DL, Dailey AT, Schmidt MH (2003) Surgical management of aneurysmal bone cysts of the spine. Neurosurg Focus 15: 13-14.

12. Chen SH, Huang TJ, Hsueh S, Lee YY, Hsu RW (2002) Unusual bleeding of aneurysmal bone cyst in the upper thoracic spine. Chang Gung Med J 25: 183-189. [Crossref]

13. O’Brien J, Ward E, Doody O, Ryan M (2009) A case of back pain associated with neurology in a young man. Ir J Med Sci 178: 373-375. [Crossref]

14. Lange T, Stehling C, Fröhlich B, Klingenhöfer M, Kunkel P, et al. (2013) Denosumab: a potential new and innovative treatment option for aneurysmal bone cysts. Eur Spine $J$ 22: 1417-1422. [Crossref]
15. Errani C, Schuster S, Biagini R, Casadei R, De Paolis M, et al. (2007) Reconstruction with fascia lata allograft of the posterior vertebra elements after resection for aneurysmal bone cyst in a child. Eur Spine J 16: 1531-1535. [Crossref]

16. Burch S, Hu S, Berven S (2008) Aneurysmal bone cysts of the spine. Neurosurg Clin NAm 19: 41-47. [Crossref]

17. Lambot-Juhan K, Pannier S, Grévent D, Péjin Z, Breton S, et al. (2012) Primary aneurysmal bone cysts in children: per-cutaneous sclerotherapy with absolute alcohol and proposal of a vascular classification. Pediatr Radiol 42: 599-605. [Crossref]

18. Simm PJ, O'Sullivan M, Zacharin MR (2013) Successful treatment of a sacral aneurysmal bone cyst with zoledronic acid. J Pediatr Orthop 33: 61-64. [Crossref]

19. Novais EN, Rose PS, Yaszemski MJ, Sim FH (2011) Aneurysmal Bone Cyst of the Cervical Spine in Children. J Bone Joint Surg Am 93: 1534-1543. [Crossref]

20. Chan MS, Wong YC, Yuen MK, Lam D (2002) Spinal aneurysmal bone cyst causing acute cord compression without vertebral collapse: CT and MRI findings. Pediatr Radiol 32: 601-604. [Crossref]

21. Varshney MK, Rastogi S, Khan SA, Trikha V (2010) Is sclerotherapy better than intralesional excision for treat-ing aneurysmal bone cysts? Clin Orthop Relat Res 468 : 1649-1659. [Crossref]

Copyright: (C2020 Osman W. This is an open-access article distributed under the terms of the Creative Commons Attribution License, which permits unrestricted use, distribution, and reproduction in any medium, provided the original author and source are credited. 